\title{
Intercambio gaseoso y eficiencia en el uso del agua de cultivos de cobertura con especies nativas (Mendoza, Argentina), exóticas cultivadas y malezas
}

\author{
Uliarte, E.M.; C.A. Parera, E.E. Alessandria y A.D. Dalmasso
}

\begin{abstract}
RESUMEN
Los cultivos de cobertura son utilizados como una herramienta ambientalmente sostenible con diversos propósitos. Una de las mayores limitantes para el uso de una cobertura vegetal es el consumo adicional de agua. Las especies herbáceas nativas adaptadas a baja disponibilidad hídrica pueden ser una alternativa factible en cultivos bajo riego localizado. El objetivo del estudio fue evaluar la eficiencia en el uso de agua y la fijación de dióxido de C de tres especies exóticas cultivadas, dos malezas y seis gramíneas nativas seleccionadas en tres regiones vitivinícolas de Mendoza, Argentina. La experimentación se efectuó en Luján de Cuyo, Mendoza, con unidades experimentales (macetas) distribuidas en diseño completamente aleatorizado con 12 tratamientos y 5 repeticiones. Se determinó consumo hídrico por el método gravimétrico e intercambio gaseoso en la planta entera. Las especies nativas tipo $\mathrm{C}_{4}$ presentaron el menor consumo hídrico anual y elevada eficiencia en el uso del agua en condiciones de restricción hídrica y temperatura elevada, debido a que su evapotranspiración se redujo proporcionalmente más que la fijación de dióxido de C. Los resultados obtenidos bajo condiciones controladas sugieren que estas gramíneas nativas pueden tener éxito como cultivos de cobertura en el sitio interfilar de viñedos bajo riego por goteo.
\end{abstract}

Palabras clave: gramíneas nativas, exóticas cultivadas, malezas, uso de agua, fijación de $\mathrm{CO}_{2}$

Uliarte, E.M.; C.A. Parera, E.E. Alessandria and A.D. Dalmasso, 2014. Gas exchange and water use efficiency of native cover crops (Mendoza, Argentina), introduced crop species and weeds. Agriscientia 31 (2): 49-61

\section{SUMMARY}

Cover crops have been largely used in the soil management of vineyards as an environmentally sustainable tool for diverse purposes. A major limitation for cover crop use is the additional water consumption. Native grasses adapted to low water availability may be a feasible alternative under drip irrigation. The aim of the study was to evaluate water use efficiency and carbon dioxide fixation of three introduced cover crops, two weeds and six native grasses 
selected from three viticultural regions of Mendoza, Argentina. The experiment was conducted in Lujan de Cuyo, Mendoza, with experimental units (pots) distributed in a completely randomized design with 12 treatments and five replicates. Water consumption following the gravimetric method and gas exchange in whole plant were measured. Native $\mathrm{C}_{4}$ grasses presented the lowest annual water consumption and high water use efficiency under elevated temperature and limited water availability, since evapotranspiration decreased proportionally more than the carbon dioxide fixation. Results obtained under controlled conditions suggest that these native grasses can succeed as interrow cover crops in vineyards under drip irrigation.

Key words: native grasses, exotic cultivated, weeds, water use, $\mathrm{CO}_{2}$ fixation

E.M. Uliarte: Instituto Nacional de Tecnología Agropecuaria (INTA), EEA Mendoza. C.A. Parera: INTA, Centro Regional Mendoza-San Juan. E.E. Alessandria: Universidad Nacional de Córdoba, Facultad de Ciencias Agropecuarias. A.D. Dalmasso: Instituto Argentino de Investigaciones de las Zonas Áridas (IADIZA), CCT CONICET Mendoza. Correspondencia a: uliarte.ernesto@inta.gob.ar

\section{INTRODUCCIÓN}

Los cultivos de cobertura se utilizan en la agricultura con diversos objetivos, entre los que se destacan el aporte de materia orgánica y nitrógeno cuando se incluyen leguminosas, el incremento en la estructura del suelo, la reducción de la compactación, el aumento en la infiltración del agua en el suelo, el control de la escorrentía y la erosión, el consumo del exceso de agua en el suelo, la mejora en la tracción de la maquinaria, el aumento de la biodiversidad, la disminución de las pérdidas de nutrientes por lixiviación, el control de malezas no deseables y la reducción en la necesidad del uso de agroquímicos (Bowman et al., 1998). Se advierte, además, que la cobertura verde puede contribuir en el secuestro de C, mediante el aporte de restos vegetales a los suelos (Sainju et al., 2002).

Específicamente en viticultura, el manejo con cobertura vegetal puede controlar el excesivo vigor de las plantas y mejorar la calidad de la cosecha, fundamentalmente en variedades tintas de vinificar (Uliarte et al., 2009b). En los viñedos argentinos, el manejo con cobertura verde es una práctica incipiente, pero que se está difundiendo progresivamente. En ese sentido, se han desarrollado en Mendoza diferentes investigaciones sobre el uso de coberturas vegetales en viñedos (Del Monte et al., 1994; Del Monte et al., 2000; Uliarte et al., 2006; Uliarte et al., 2009a,b).

Entre las desventajas del uso de un cultivo de cobertura se pueden citar el incremento del riesgo de heladas (Donaldson et al., 1993) y la competencia por nutrientes (Celette et al., 2009), la cual en caso de ser demasiado fuerte puede causar defectos aromáticos en vinos blancos (Maigre et al., 1995).

No obstante, uno de los mayores perjuicios corresponde al consumo adicional de agua en el viñedo (Monteiro \& Lopes, 2007), debido a la creciente preocupación por reducir el uso del recurso en la agricultura irrigada (Boutraa, 2010). Se han encontrado diferencias en el consumo hídrico según la especie de cobertura utilizada. Estudios al respecto, demostraron la gran variabilidad en las tasas de transpiración de algunas malezas y cultivos de cobertura presentes en los viñedos (Lopes et al., 2004). El consumo de agua es un tema crítico para la elección de las coberturas vegetales (Olmstead et al., 2001; Lopes et al., 2004; Spring \& Delabays, 2006). Se sabe, asimismo, que la eficiencia en el uso del agua de las especies varía de acuerdo al estado hídrico del suelo (Singh \& Misra, 1985). Por lo tanto, la especie de cobertura vegetal a utilizar y su manejo están condicionados fuertemente por la disponibilidad hídrica, factor limitante en los ecosistemas áridos y semiáridos de los valles cultivados de Mendoza.

La incorporación de cultivos de cobertura conlleva a un mayor consumo de agua, por lo que su implementación en regiones con restricciones hídricas puede resultar inconveniente; de ahí que su manejo debe ajustarse a promover la utilización más eficiente del agua. Prichard et al. (1989) encontraron que el consumo de agua en cultivos de almendros con riego por aspersión del Valle Central de California (EE.UU.), fue entre 10 y 30\% mayor bajo condiciones de cobertura vegetal permanente que cuando el cultivo fue tratado con herbicida. Mientras que en otro estudio, efectuado también 
en California, se determinó que el incremento de la infiltración del agua debido al uso de coberturas permanentes en un viñedo, aumentó igualmente el uso de agua en un 46\% en comparación con el suelo descubierto (Gulick et al., 1994). Yunusa et al. (1997) calcularon la evapotranspiración (Evt) total para un viñedo con riego superficial en el sudeste de Australia, y encontraron que la Evt correspondiente a la cobertura verde fue de 30 a $36 \%$ del total. En un viñedo de secano con clima mediterráneo (región de Extremadura, Portugal) se estimó que un suelo con cobertura vegetal consume hasta un 9\% más de agua, en comparación con el suelo labrado. Este último valor debe interpretarse con cuidado, ya que el estudio tomó en cuenta el contenido hídrico solo hasta $1 \mathrm{~m}$ de profundidad, por lo que se sugiere que la proporción real sería muy superior (Monteiro \& Lopes, 2007). Recientemente, una investigación realizada en una zona de mayor humedad (Nueva York, EE.UU.) calculó que entre 27 y $31 \%$ de la Evt total de un viñedo correspondió a la cobertura verde perenne (Centinari et al., 2012). En la zona alta del río Mendoza se comprobó que para regar un viñedo mantenido con cobertura vegetal permanente y regado superficialmente por melga, fue necesario un volumen de agua entre 44 y $86 \%$ mayor ( 21 y $42 \%$ cuando se utilizaron especies anuales), respecto de suelo descubierto con aplicación de herbicida. Estas notables diferencias fueron atribuidas no tanto al incremento en el consumo hídrico, sino más bien a la considerable reducción del frente de avance del agua de riego en la melga, por parte de la cobertura verde y los restos vegetales, lo cual redujo la eficiencia del riego (Uliarte \& Del Monte, 2006). En estos casos la eficiencia puede mejorarse mediante la formación periódica de surcos superficiales de avance, a ambos lados de la melga.

La interacción entre la cobertura vegetal y un cultivo perenne puede determinar un aumento en el uso del agua, respecto del suelo descubierto, este incremento dependerá de diversos factores tales como las especies utilizadas, el grado de cobertura del suelo, el sistema de riego o las características edafo-climáticas de cada sitio.

Resulta fundamental profundizar en la cuantificación del consumo de agua y la eficiencia de su uso por parte de diferentes coberturas.

Ante el incremento en la temperatura y el déficit hídrico previstos por diferentes modelos de cambio climático para la región centro-oeste de la Argentina (Hulme \& Sheard, 1999), las especies nativas, mejor adaptadas a las condiciones regionales e íntimamente ligadas al uso eficiente del agua, ofrecen ventajas comparativas para ser utilizadas como cultivo de cobertura en viñedos manejados con riego localizado (Penfold, 2010).

El objetivo del trabajo fue comparar la eficiencia en el uso del agua de cultivos de cobertura nativos y especies introducidas a nivel del sistema planta entera-suelo y medir su potencial fijación de dióxido de C.

\section{MATERIALES Y MÉTODOS}

\section{Selección de las especies bajo estudio}

En marzo del año 2007 se analizó la composición específica de la cobertura vegetal espontánea, favorecida mediante segados periódicos, en tres viñedos seleccionados de diferentes zonas vitícolas de la provincia de Mendoza (Tabla 1), los cuales tienen la particularidad de poseer riego por goteo y haber sido implantados en suelo virgen previo desmonte de la vegetación nativa. Utilizando el método de la cuerda con cuentas (Sarrantonio, 1995) se detectaron seis especies herbáceas dominantes (gramíneas), dos en cada sitio de observación, las cuales en conjunto representaron no menos del $85 \%$ del total de especies para cada sitio.

Tabla 1. Descripción de los sitios de seguimiento donde se seleccionó la vegetación nativa dominante.

\begin{tabular}{lccc}
\hline Viñedo & Finca Clement & Fincas del Inca & Finca La Celia \\
\hline Ubicación & Santa Rosa & Maipú & San Carlos \\
& (Alto Verde) & (Barrancas) & (Eugenio Bustos) \\
Latitud & $33^{\circ} 07^{\prime} \mathrm{S}$ & $33^{\circ} 04^{\prime} \mathrm{S}$ & $33^{\circ} 46^{\prime} \mathrm{S}$ \\
Longitud & $68^{\circ} 11^{\prime} \mathrm{O}$ & $68^{\circ} 44^{\prime} \mathrm{O}$ & $69^{\circ} 07^{\prime} \mathrm{O}$ \\
Altitud (msnm) & 622 & 789 & 1.020 \\
Zona vitivinícola (1) & Este mendocino & Zona alta del río Mendoza & Valle de Uco \\
Clima vitícola (1) & Caluroso, con sequía fuerte & Caluroso, con sequía & Caluroso, con sequía \\
& y noches templadas & moderada y noches frías & moderada y noches muy frías \\
\hline Tipo de suelo (2) & Arenoso - franco & Arenoso - franco & Franco - arenoso \\
\hline
\end{tabular}

Abreviatura: m.s.n.m.: metros sobre el nivel del mar.

Referencias: (1) según Catania et al. (2007). (2) Clasificación textural americana. 
Cinco gramíneas nativas tipo $\mathrm{C}_{4}$ (Digitaria californica (Benth.) Henrard, Leptochloa dubia (Kunth) Nees, Setaria mendocina Phil., Pappophorum caespitosum R.E. Fr., Sporobolus cryptandrus (Torr.) A. Gray) y una gramínea nativa tipo $\mathrm{C}_{3}$ (Nassella tenuis (Phil.) Barkworth) fueron las especies dominantes seleccionadas para comparar su comportamiento con tres especies exóticas cultivadas; una leguminosa (Trifolium repens L.) y una gramínea (Festuca arundinacea Schreb.), ambas perennes tipo $\mathrm{C}_{3}$; una gramínea anual invernal $\mathrm{C}_{3}$ (Secale cereale L.); y dos malezas perennes de actividad estival (tipo $\mathrm{C}_{4}$ ) de amplia difusión (Sorghum halepense (L.) Pers. y Cynodon dactylon (L.) Pers.).

Para análisis posteriores, las especies fueron agrupadas según sus atributos funcionales y origen; así quedaron conformados los diferentes grupos: nativas $\mathrm{C}_{4}$ (perennes de actividad estival), nativa $\mathrm{C}_{3}$ (perenne de actividad invernal), cultivadas perennes $\mathrm{C}_{3}$, cultivada invernal $\mathrm{C}_{3}$, malezas estivales $\mathrm{C}_{4}$.

\section{Sitio y diseño experimental}

El ensayo se desarrolló en la EEA Mendoza del INTA, ubicada en Luján de Cuyo, Mendoza (3300'21" S, 6851'53" O, 929 msnm). Los datos meteorológicos del período de experimentación se presentan en la Figura 1.

Las unidades experimentales (macetas) fueron distribuidas en diseño completamente al azar con once tratamientos de diferentes cultivos de cobertura, un testigo con suelo descubierto y cinco repeticiones por tratamiento. Las especies perennes fueron establecidas en el mes de setiembre de 2007 mediante trasplante, mientras que la única anual ( $S$. cereale) fue sembrada en otoño de 2008 y 2009.

En macetas plásticas de 30 litros de capacidad (diámetro: 0,40 m; altura: 0,34 m) conteniendo un sustrato compuesto por $60 \%$ de turba, $30 \%$ de perlita (granulado volcánico) y 10\% de tierra de embanque (franco-arenosa, libre de malezas), se implantaron tres individuos adultos de cada especie perenne por maceta, a fin de obtener en pocos meses una alta proporción del área cubierta con cada cultivo de cobertura. Para la especie anual, donde se preveía un bajo nivel de cobertura durante su establecimiento, se elevó la densidad de plantas a entre 6 a 12 plantas por maceta, provenientes de semilla. Macetas con el mismo sustrato fueron mantenidas libres de vegetación como tratamiento testigo (suelo descubierto). Cada maceta recibió 15 litros de agua de pozo por cada riego, en forma de lluvia mediante regadera, con una frecuencia de 7 a 21 días, según ensayo y época del

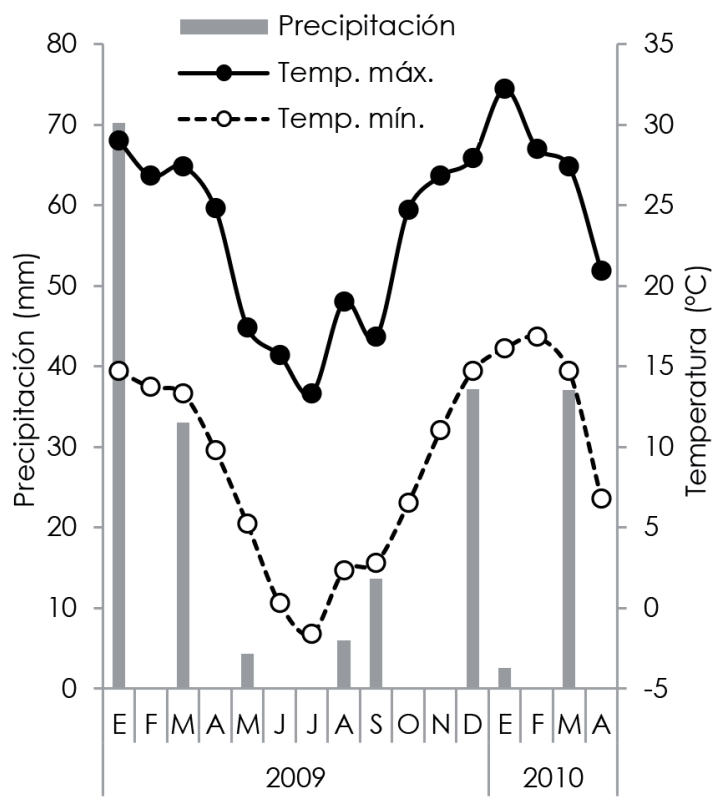

Figura 1. Datos meteorológicos del sitio de experimentación, en el transcurso del período de duración del ensayo (estación meteorológica "Chacras de Coria"). A partir de datos provistos por la Facultad de Ciencias Agrarias de la Universidad Nacional de Cuyo.

año. En el verano de 2009 se definió una frecuencia de 12 días para estudiar el comportamiento de las especies bajo condiciones de restricción hídrica, mientras que en el verano de 2010 se efectuó un riego cada 7 días para evaluar las especies en condiciones de alta disponibilidad de agua. En otoño, invierno y primavera de 2009 se definió una frecuencia de riego de 11, 21 y 10 días respectivamente, sin limitación de agua.

\section{Consumo hídrico y mediciones de intercambio gaseoso}

Se realizó una estimación del consumo de agua de las diferentes coberturas vegetales siguiendo el método gravimétrico, registrando periódicamente la pérdida de peso de las macetas después de un riego, mediante una balanza digital (Leal XXI, Tecnel, Mendoza, Argentina). Este análisis asume como despreciable la potencial contribución de peso dada por el desarrollo vegetativo de las plantas entre pesadas. El contenido hídrico en el sustrato se expresó como porcentaje, respecto del contenido de agua en capacidad de campo (100\%), denominándose "contenido relativo de agua en el sustrato" (Cras). La capacidad de cam- 
po se definió a partir del peso de las macetas 24 horas después de un riego. Se calculó la Evt media diaria por conjunto de especies, empleando el promedio de las especies incluidas en cada grupo.

Las mediciones de intercambio gaseoso se efectuaron mediante un sistema de cámara abierta para canopia (CPY-3, PP Systems, Hitchin, Reino Unido), modificado en el Instituto de Investigación de Geisenheim (Alemania) para ser usado con cámaras cilíndricas de 0,50 m de diámetro y 0,50 m de altura con un volumen total de 0,098 $\mathrm{m}^{3}$. La cámara fue equipada con un ventilador próximo a un termistor (NTC), utilizado para determinar la temperatura del aire dentro de la cámara. La radiación de fotones fotosintéticamente activos (RFA) dentro de la cámara fue medida con un sensor puntual quántico (EMS 7, PP Systems, Hitchin, Reino Unido). Se verificó que la penetración de la luz dentro de la cámara, determinada con un espectro-radiómetro (modelo 100-40, Hitachi, Japón), fue levemente atenuada (alrededor del 15\%) en la región de los 300 a 1000 nm, debido al film de poliéster (Hostaphan, Pütz, Frankfurt, Alemania) utilizado como cobertor de la cámara.

El monitoreo de gases se realizó mediante un analizador infrarrojo de gases (EGM4, PP Systems, Hitchin, Reino Unido). Los flujos de dióxido de C y de agua de la canopia fueron medidos como diferencias en sus fracciones molares, entre la muestra de gas de referencia tomada en la entrada de aire y la medición de gas de la muestra tomada en el interior de la cámara.

Durante las mediciones, el sistema trabajó con un suministro de aire de 50 a 65 I min-1, valor ligeramente inferior a lo sugerido por el fabricante del equipo (PP System) (70 a $90 \mid \mathrm{min}^{-1}$ ), esto fue debido a una limitación presentada por el regulador de caudal de aire. Dependiendo de las condiciones externas, la temperatura del aire dentro de la cámara presentó una desviación de $+2 \mathrm{a}+5^{\circ} \mathrm{C}$ respecto de la temperatura exterior.

Se efectuaron dos tipos de mediciones:

1- Intercambio gaseoso determinado a mediodía (entre las 11:00 y 15:00 h), en las diferentes estaciones del año. El equipo tomó datos automáticamente cada minuto durante un período de 5 minutos, antes de ser trasladado a la siguiente maceta. Se midió en condiciones adecuadas de humedad de suelo y con restricción hídrica (2 y 8 días después de regar, respectivamente).

2- Dinámica diaria del intercambio gaseoso, monitoreada en cinco momentos del día (preamanecer, media-mañana, mediodía, media-tarde y luego de la puesta del sol), en las cuatro estaciones del año. El equipo registró mediciones cada minuto durante 3 minutos, antes de ser trasladado a la siguiente maceta. En este caso se seleccionaron tres tratamientos con cultivos de cobertura ( $F$. arundinacea, $N$. tenuis y $D$. californica) y el testigo con suelo descubierto teniendo en cuenta solo tres repeticiones, debido al tiempo demandado para efectuar las mediciones. Utilizando datos de seguimiento de la dinámica diaria del intercambio gaseoso (datos no presentados) y efectuando interpolación para cada hora, se estimó el balance diario de Evt y de dióxido de C.

Valores negativos en las tasas de balance de dióxido de $\mathrm{C}$ indicaron asimilación o fijación $(A)$, mientras que valores positivos correspondieron a emisión o liberación de $\mathrm{C}$ a la atmósfera $(E)$.

De acuerdo a las calibraciones efectuadas y teniendo en cuenta el caudal de aire de trabajo utilizado (50 a 65 I min-1), los valores de evapotranspiración (Evt) calculados por el analizador de gases, para demandas evaporativas elevadas, fueron del $70 \%$ respecto de los determinados a través de la pérdida de peso en balanza. Estas diferencias pueden atribuirse a desviaciones en la calibración del sensor de humedad del sistema y el insuficiente caudal de aire de trabajo; de todas formas, puesto que fueron estables en el tiempo, siguen siendo datos útiles para la comparación de las diferentes especies con respecto a su uso relativo de agua. A los datos de Evt se les aplicó un factor de corrección de $+30 \%$, por la subestimación observada en comparación con el método gravimétrico.

\section{Técnicas estadísticas}

Se realizó análisis de la varianza y de contrastes ortogonales para comparar grupos de especies mediante el software Infostat versión 2011 (Di Rienzo et al., 2011). Las diferencias entre las medias de los tratamientos fueron comparadas empleando la prueba de Tukey $(p \leq 0,05)$. Se determinaron los errores estándares para las medias de los tratamientos, los cuales aparecen en figuras y tablas. Los gráficos fueron creados con el software Sigmaplot 11.0 (Systat Software Inc., 2008) y Excel 2010 (Microsoft Corporation, 2010).

\section{RESULTADOS}

\section{Consumo hídrico y Evt de diferentes cultivos de cobertura (método gravimétrico)}

La Figura 2 muestra la evolución en el año para cada uno de estos grupos y en la Tabla 2 se presenta un análisis de contrastes ortogonales para 
compararlos entre sí.

Para el verano de 2009 con restricción hídrica, el grupo de nativas $\mathrm{C}_{4}$ tuvo un consumo significativamente mayor al resto de los tratamientos, mientras que el de la nativa $\mathrm{C}_{3}$ fue similar al de exóticas cultivadas perennes $\mathrm{C}_{3}$ y malezas $\mathrm{C}_{4}$.

En otoño sin limitación de agua, todas redujeron su Evt, aunque en mayor proporción lo hicieron las nativas y malezas $\mathrm{C}_{4}$, sin diferenciarse estadísticamente de la evaporación del suelo descubierto. Las exóticas cultivadas perennes $\mathrm{C}_{3}$ mantuvieron un consumo significativamente superior al resto, debido a su prolongado ciclo vegetativo en otoño.

Durante el invierno sin restricción hídrica, como era de esperar, la Evt continuó descendiendo para todas las especies, pero de manera más marcada aún en las nativas y malezas $\mathrm{C}_{4}$, siendo incluso considerablemente inferior a la evaporación del suelo descubierto.

A partir de la primavera, sin limitación de agua el consumo de las especies aumentó de forma notable, destacándose las malezas $\mathrm{C}_{4}$ y las exóticas cultivadas $\mathrm{C}_{3}$ (perenne e invernal) con valores significativamente superiores a las nativas $\mathrm{C}_{4}$. La de menor consumo primaveral resultó ser la nativa $\mathrm{C}_{3}$.

En el verano de 2010 con riegos frecuentes se

Tabla 2. Análisis de contrastes ortogonales para la Evt media diaria por grupo de especie y para diferentes épocas del período 2009-2010.

\begin{tabular}{|c|c|c|}
\hline & & $\begin{array}{c}\text { Nivel de } \\
\text { significancia } \\
\text { (valor de } \rho \text { ) }\end{array}$ \\
\hline \multirow[b]{2}{*}{$\begin{array}{l}\text { Verano } \\
2009\end{array}$} & Nativa $\mathrm{C}_{4}$ vs. resto & $<0,0001^{\star \star}$ \\
\hline & $\begin{array}{l}\text { Nativa } \mathrm{C}_{3} \text { vs. cultivada per. } \\
\mathrm{C}_{3}+{\text { maleza } \mathrm{C}_{4}}\end{array}$ & $0,7487 \mathrm{~ns}$ \\
\hline \multirow{2}{*}{$\begin{array}{l}\text { Otoño } \\
2009\end{array}$} & $\begin{array}{l}\text { Nativa } \mathrm{C}_{4}+\text { maleza } \mathrm{C}_{4} \text { vs. } \\
\text { suelo }\end{array}$ & $0,1286 \mathrm{~ns}$ \\
\hline & Cultivada per. $\mathrm{C}_{3}$ vs. Resto & $<0,0001^{\star \star}$ \\
\hline $\begin{array}{l}\text { Invierno } \\
2009\end{array}$ & $\begin{array}{l}\text { Nativa y maleza } \mathrm{C}_{4} \text { vs. } \\
\text { Suelo }\end{array}$ & $<0,0001$ ** \\
\hline \multirow{2}{*}{$\begin{array}{l}\text { Primavera } \\
2009\end{array}$} & $\begin{array}{l}\text { Maleza } C_{4}+\text { cultivada (per. } \\
\text { e inv.) } C_{3} \text { vs. nativa } C_{4}\end{array}$ & 0,0004 ** \\
\hline & Nativa $\mathrm{C}_{3}$ vs resto & 0,0003 ** \\
\hline \multirow{2}{*}{$\begin{array}{l}\text { Verano } \\
2010\end{array}$} & $\begin{array}{l}\text { Cultivada per. } \mathrm{C}_{3} \text { vs. resto } \\
\left(\sin \text { inv. } \mathrm{C}_{3}\right)\end{array}$ & $<0,0001^{* *}$ \\
\hline & Nativa $\mathrm{C}_{4}$ vs maleza $\mathrm{C}_{4}$ & 0,0039 ** \\
\hline
\end{tabular}

ns: diferencia no significativa.

**: diferencia altamente significativa $(\rho \leq 0,01 ; n=5)$.

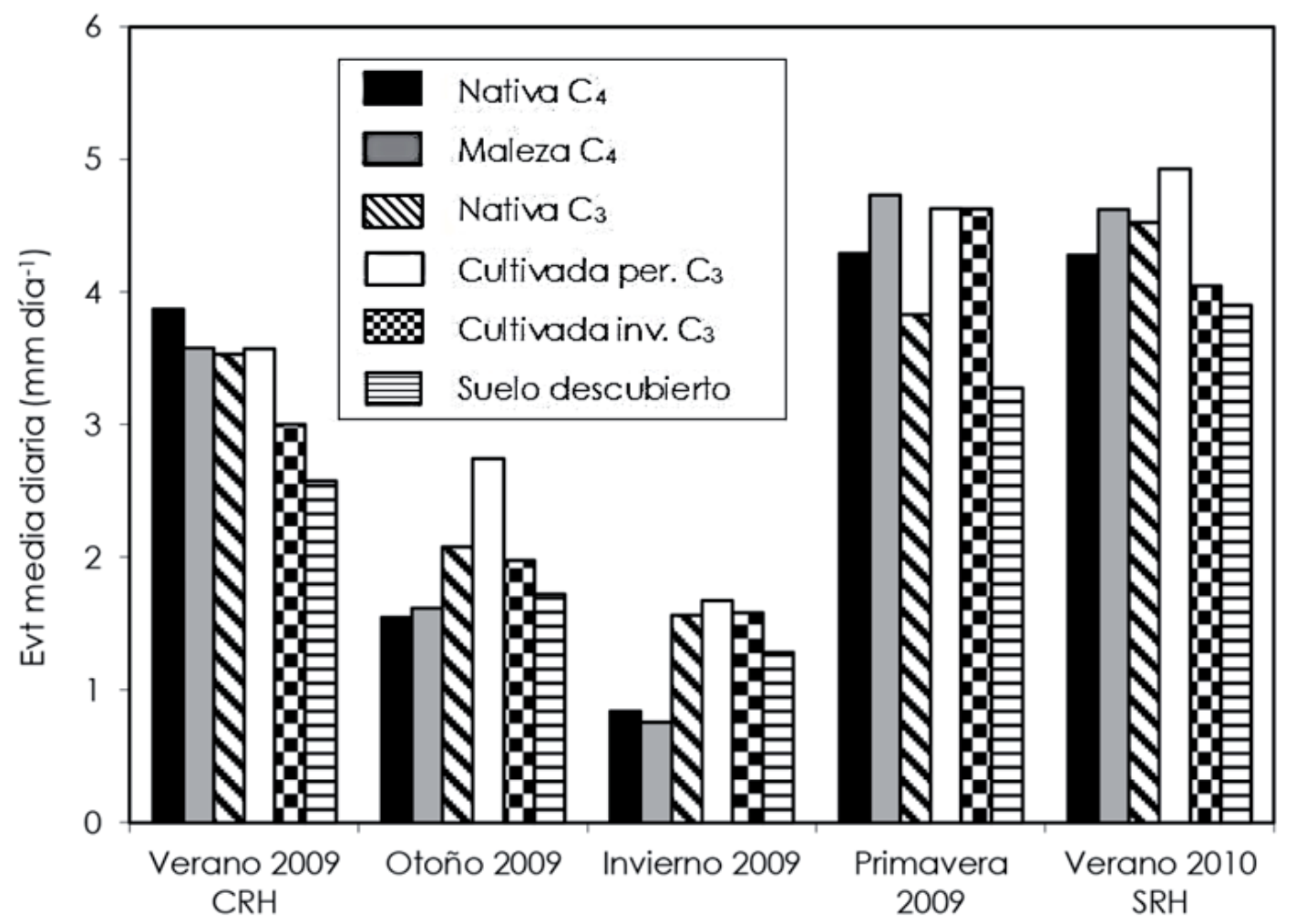

Figura 2. Evt media diaria por grupo de especie y para diferentes épocas del período 2009-2010. CRH: con restricción hídrica; SRH: sin restricción hídrica. 
observaron dos comportamientos diferentes. Por un lado los grupos de nativa $\mathrm{C}_{3}$ y cultivada perenne $\mathrm{C}_{3}$, quienes aumentaron su Evt respecto de la primavera; por otra parte, las nativas $\mathrm{C}_{4}$ y malezas $\mathrm{C}_{4}$, mantuvieron un consumo muy próximo al primaveral. Dentro de los grupos de especies que permanecieron activas durante el verano, las exóticas cultivadas perennes $\mathrm{C}_{3}$ presentaron un consumo significativamente superior al resto, mientras que las nativas $\mathrm{C}_{4}$ mostraron una Evt marcadamente inferior a la de las malezas $\mathrm{C}_{4}$.

Al comparar el comportamiento de la Evt para los diferentes grupos de especies, entre un verano (i.e., considerando los meses de enero, febrero y marzo) con riegos frecuentes y otro con cierta restricción hídrica, se observó, para todos los tratamientos, una reducción en el consumo de agua. Para las especies nativas del tipo $\mathrm{C}_{4}$ esta disminución fue de $9,5 \%$, mientras que para el resto estuvo entre 22 y $27 \%$, por lo que de poseer un consumo de agua relativamente bajo (verano sin restricción), pasó a ser el grupo de mayor consumo bajo condiciones de estrés. En situación de baja disponibilidad hídrica, los tres grupos citados anteriormente pasaron a tener un consumo casi idéntico. Un caso para analizar aparte es la cultivada $\mathrm{C}_{3}$ (i.e., $S$. cereale), ya que se trató de una especie inactiva durante el verano y que fue resembrada a fines del verano o a principios de otoño, por lo que su Evt fue baja y próxima a la evaporación del suelo descubierto (i.e., testigo) para las dos situaciones. Por otro lado, el testigo resultó ser el tratamiento con mayor reducción en el gasto hídrico, presentando una disminución de la evaporación de 34\%.

Al analizar el consumo hídrico anual (Figura 3), se apreciaron valores que fueron desde los $868 \mathrm{~mm}$ (suelo descubierto) hasta los $1212 \mathrm{~mm}$ (cultivada perenne $\mathrm{C}_{3}$ ); se puede notar que todas las coberturas vegetales generaron un aumento respecto de la evaporación alcanzada con el suelo descubierto (valor de referencia). Las especies nativas del tipo $\mathrm{C}_{4}$ fueron mayores en un $13 \%$, mientras que las perennes exóticas del tipo $\mathrm{C}_{3}$ presentaron un aumento de hasta $40 \%$. El resto de las especies se mantuvo en un consumo anual de entre 18 y $23 \%$ superior al de un suelo descubierto.

\section{Intercambio gaseoso y eficiencia en el uso del agua durante el mediodía}

Evapotranspiración (Evt). Durante un día de verano con elevado contenido de humedad en el sustrato, las especies de mayor Evt fueron C. dactylon y $F$. arundinacea, aunque las diferencias solo resultaron significativas respecto de S. cereale, S.

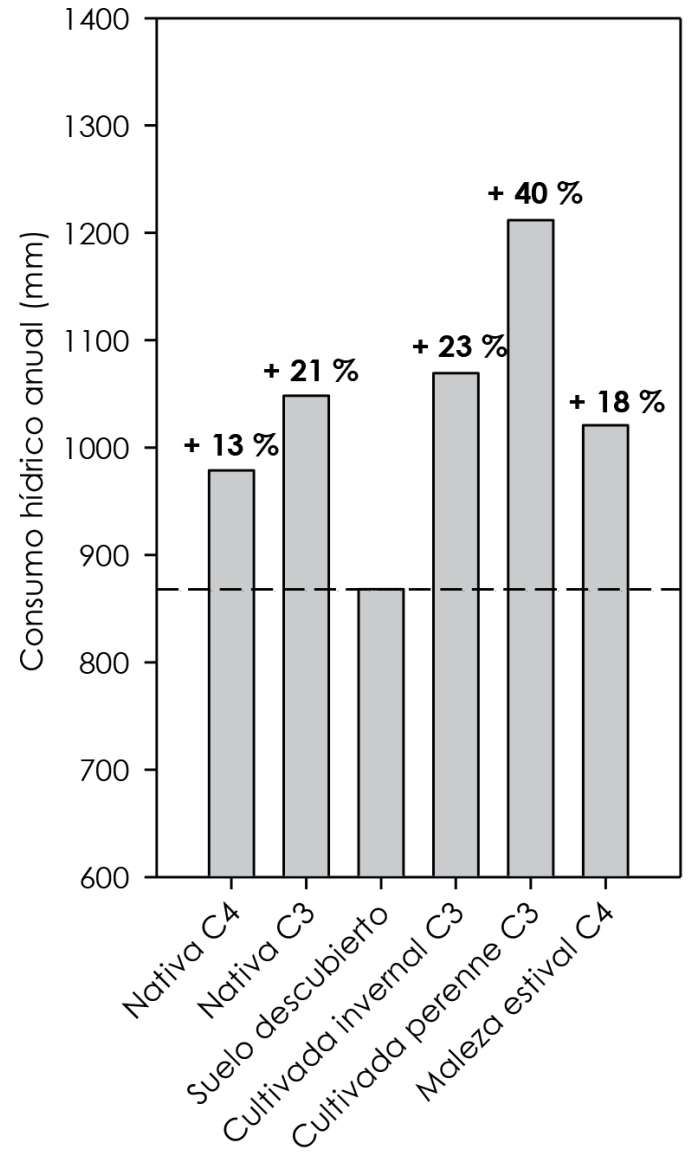

Figura 3. Estimación del consumo hídrico anual por grupo de especie, determinado por gravimetría en macetas durante el año 2009 y los tres primeros meses de 2010, y porcentaje de aumento en el consumo respecto del testigo. El valor de referencia está dado por la evaporación del suelo descubierto.

mendocina y $P$. caespitosum. Estas dos últimas especies nativas tipo $\mathrm{C}_{4}$ presentaron valores de Evt similares a los de evaporación en el tratamiento de $S$. cereale (como rastrojo por finalización de su ciclo). Los tres tratamientos mencionados exhibieron tasas de Evt significativamente inferiores a la evaporación del suelo descubierto (Figura 4A). Ocho días después del riego la Evt se redujo notablemente en todos los tratamientos (Figura 4D). Bajo estas condiciones la especie con mayor tasa de Evt resultó ser $C$. dactylon, aunque sin mostrar diferencias respecto de las nativas (i.e., S. mendocina, P. caespitosum, D. californica, L. dubia, S. cryptandrus y $N$. tenuis) y de $S$. halepense. Mientras que la evaporación del rastrojo de $S$. cereale y la Evt de $T$. repens, no se diferenciaron del testigo con suelo descubierto. 
Balance de C. Las mayores tasas de $A$ durante un día caluroso y elevado contenido de humedad en el sustrato pertenecieron a $C$. dactylon, quien se diferenció significativamente del resto de las especies, con la excepción de la nativa $D$. californica. Las menores tasas de fijación de dióxido de $\mathrm{C}$ correspondieron a F. arundinacea y $\mathrm{N}$. tenuis ( $\mathrm{Fi}-$ gura 4B). En condiciones de restricción hídrica, las especies nativas y malezas del tipo $\mathrm{C}_{4}$ redujeron sus tasas de $A$, aunque manteniendo el balance de fijación de C. Mientras tanto las perennes exóticas (i.e., $T$. repens y $F$. arundinacea) y la nativa, todas del tipo $\mathrm{C}_{3}$, mostraron tasas de $\mathrm{E}$ de dióxido de $\mathrm{C}$ al igual que $S$. cereale y el testigo (Figura 4E).

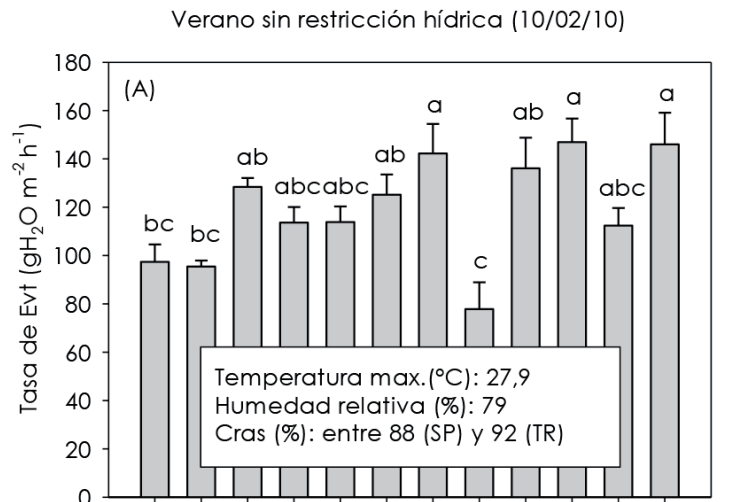

Verano con restricción hídrica (18/02/10)
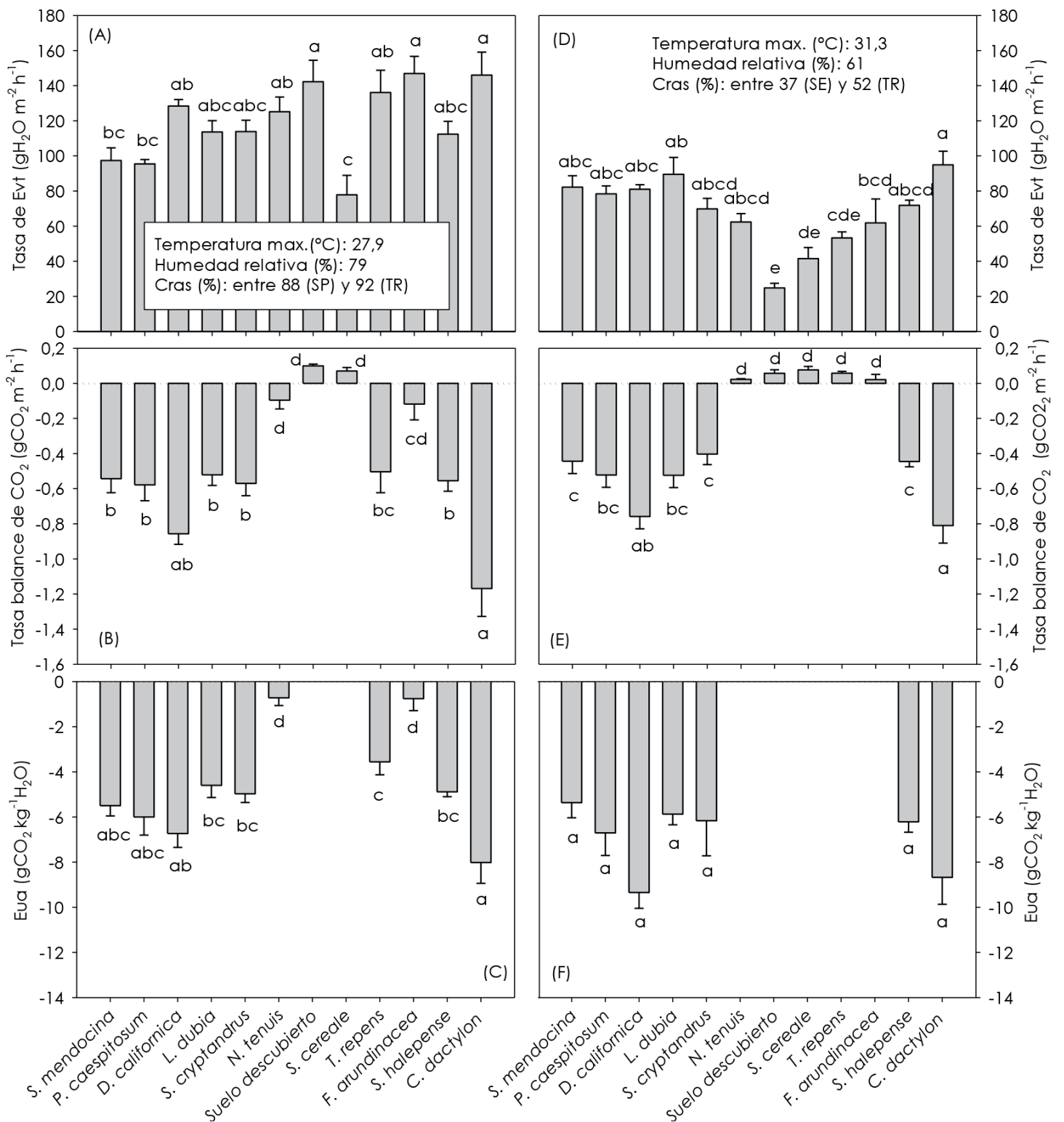

Figura 4. Tasas de Evt ( $\mathrm{A}$ y D), A (B y E) y Eua ( $\mathrm{C}$ y F) para diferentes especies de cobertura vegetal durante febrero de 2010. Los gráficos $A, B$ y $C$ presentan valores obtenidos durante un período sin restricción hídrica, mientras que los gráficos $D$, E y $F$ durante una etapa con limitación de agua. Mediciones efectuadas entre las 11 y 15 hora local. Letras distintas indican diferencias estadísticas significativas entre las medias (prueba de Tukey; $\rho \leq 0,05 ; n=4$ ), mientras que las líneas verticales muestran el error estándar. Cras: contenido relativo de agua en el sustrato. 
Eficiencia en uso del agua (Eua). En una jornada de verano, en condición de alto contenido hídrico en el sustrato, el tratamiento de $C$. dactylon se destacó por una mayor Eua, aunque sin diferenciarse estadísticamente de tres de las nativas $\mathrm{C}_{4}$ (i.e., S. mendocina, P. caespitosum y D. californica). Las menores eficiencias correspondieron a especies $\mathrm{C}_{3}$ (i.e., N. tenuis y $F$. arundinacea) (Figura 4C). En situación de restricción hídrica, no se detectaron diferencias entre la Eua de nativas y malezas $\mathrm{C}_{4}$; se observó en general que los valores se mantuvieron o bien se incrementaron levemente, mientras que para las especies $\mathrm{C}_{3}$ se redujo hasta cero (Figura 4C y F).

Relación entre $\boldsymbol{A}$ y Eua. Se analizó la relación entre la tasa de $A$ y la Eua, para malezas y nativas tipo $\mathrm{C}_{4}$, bajo dos condiciones de humedad en el sustrato. No se detectaron diferencias de Eua entre malezas y nativas $C_{4}$, pero sí al diferenciar su estado hídrico. Se comprobó que para tasas de $A$ de entre $-0,5$ y $-1,0 \mathrm{~g} \mathrm{CO}_{2} \mathrm{~m}^{-2} \mathrm{~h}^{-1}$, la Eua fue superior en situaciones de restricción de agua. Con sustrato seco y valores de $A$ de $-1,0 \mathrm{~g} \mathrm{CO}_{2} \mathrm{~m}^{-2} \mathrm{~h}^{-1}$, se alcanzaron valores máximos de Eua cercanos $\mathrm{a}-11 \mathrm{~g} \mathrm{CO}_{2} \mathrm{~kg}^{-1} \mathrm{H}_{2} \mathrm{O}$. Para conseguir ese valor de eficiencia en sustrato húmedo se requirieron tasas de fijación superiores a $-2,5 \mathrm{~g} \mathrm{CO}_{2} \mathrm{~m}^{-2} \mathrm{~h}^{-1}$. Como era de esperar, en condiciones de sustrato húmedo se alcanzaron mayores niveles de fijación de C (Figura 5).

\section{Estimación del balance diario de intercambio gaseoso}

Durante un día con temperaturas promedio de febrero (i.e., temperatura máxima de $28,9{ }^{\circ} \mathrm{C}$ ) y buena humedad en el sustrato (i.e., Cras: 81 a $83 \%)$, se calculó para las coberturas vegetales una
Evt de entre 1,52 y $1,80 \mathrm{~mm}$ día ${ }^{-1}$ (entre un 20 y $43 \%$ superior al suelo descubierto). El tratamiento de $D$. californica presentó una Evt intermedia (i.e., 1,72 $\mathrm{mm}$ día $\left.{ }^{-1}\right)$, siendo la única con balance positivo de $A$ de dióxido de $\mathrm{C}$ (más de $-4 \mathrm{~g} \mathrm{CO}_{2} \mathrm{~m}^{-2}$ día ${ }^{-1}$ ). Las dos especies $\mathrm{C}_{3}$ mostraron valores positivos de $E$ de dióxido de $\mathrm{C}$, siendo superiores a los de la respiración del testigo con suelo descubierto (Tabla 3).

\section{DISCUSIÓN}

\section{Consumo hídrico de las coberturas vegetales}

Se estimó un aumento del consumo hídrico anual promedio de $40 \%$, por parte de una especie cultivada perenne $\mathrm{C}_{3}$, respecto del suelo descubierto, valor que se corresponde con los antece-

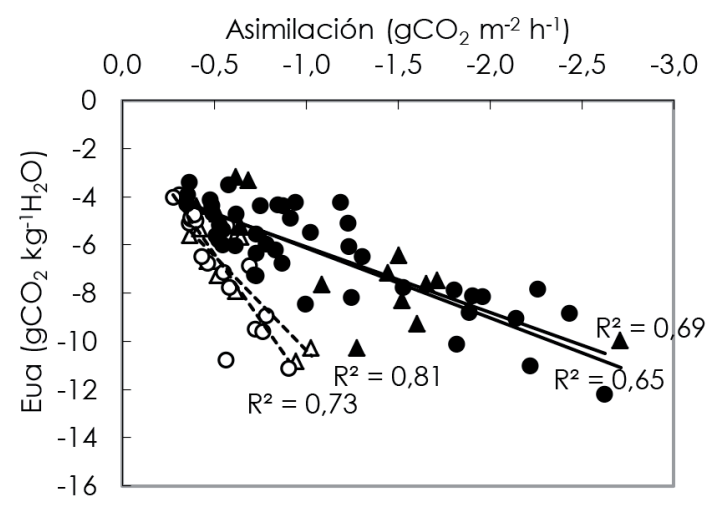

Figura 5. Relación entre la tasa de A y la Eua en especies tipo C4, bajo diferentes condiciones de humedad de suelo. Mediciones efectuadas en febrero y marzo de 2009 y 2010 , entre las 11 y 15 hora local. Los círculos representan las especies nativas $\mathrm{C} 4$ y los triángulos las malezas $\mathrm{C} 4$, mientras que los símbolos en negro indican el sustrato húmedo y en blanco el sustrato seco. Para cada curva se indica el grado de relación entre las variables.

Tabla 3. Estimación del balance diario de Evt y dióxido de $C$ para diferentes especies de cobertura vegetal.

\begin{tabular}{|c|c|c|c|c|}
\hline \multicolumn{5}{|c|}{ Fecha: $11 / 02 / 10$} \\
\hline & & nperatura máxima $\left({ }^{\circ} \mathrm{C}\right)$ & 28,9 & \\
\hline & & mperatura mínima $\left({ }^{\circ} \mathrm{C}\right)$ & 25,8 & \\
\hline & Evt potencial (Penm & an-Monteith) (mm día $\left.{ }^{-1}\right)$ & 3,2 & \\
\hline Tratamiento & $\begin{array}{l}\text { Cras } \\
(1)(\%)\end{array}$ & $\begin{array}{l}\text { Tasa diaria de Evt (2) } \\
\qquad\left(\mathrm{mm} \mathrm{dia}^{-1}\right)\end{array}$ & $\begin{array}{c}\text { Aumento de consumo } \\
\text { respecto de la } \\
\text { referencia (\%) }\end{array}$ & $\begin{array}{c}\text { Tasa diaria balance de C } \\
(2)(3) \\
\left(\mathrm{g} \mathrm{CO}_{2} \mathrm{~m}^{-2} \text { día }^{-1}\right)\end{array}$ \\
\hline D. californica & 83 & 1,72 & 37 & $-4,07$ \\
\hline N. tenuis & 82 & 1,52 & 20 & 4,55 \\
\hline F. arundinacea & 80 & 1,80 & 43 & 4,92 \\
\hline Suelo descubierto & 81 & 1,26 & referencia & 2,06 \\
\hline
\end{tabular}

(1) Cras: contenido relativo de agua en el sustrato.

(2) Mediciones efectuadas cada cuatro horas en el transcurso del día, los valores horarios restantes se obtuvieron mediante interpolación.

(3) Valores negativos en la tasa diaria de balance de $C$ indican fijación $(A)$, mientras que valores positivos expresan emisión (E). 
dentes citados en la bibliografía. Con la utilización de especies anuales, estivales e invernales, el incremento fue de entre 18 y $23 \%$, respectivamente. Mientras que mediante el uso de nativas el valor se redujo a $21 \%$ (en tipo $\mathrm{C}_{3}$ ) y $13 \%$ (en tipo $\mathrm{C}_{4}$ ), lo cual permite conjeturar que a través del manejo de coberturas herbáceas nativas se podría reducir el consumo de agua hasta un $27 \%$, en cultivos perennes manejados con cobertura verde permanente. Estos valores anuales, estimados por gravimetría, fueron confirmados con mediciones de intercambio gaseoso efectuadas a mediodía; allí se determinaron aumentos en el consumo hídrico diario de entre 20 y $43 \%$, por parte de las coberturas y comparadas con el suelo descubierto.

Cabe aclarar que existe el antecedente del intento de establecer especies herbáceas nativas en viñedos bajo riego superficial (melga), el cual no prosperó debido a la gran capacidad de competencia de las malezas introducidas, las que bajo condiciones de elevada disponibilidad hídrica terminaron predominando por sobre las especies nativas (Cavagnaro \& Dalmasso, 1986).

En cultivos provistos con riego por goteo resulta complicado establecer las especies introducidas comúnmente utilizadas como coberturas verdes, salvo bajo ciertas condiciones particulares, tales como cuando se trabaja, por ejemplo, utilizando especies con relativa tolerancia a la sequía, en suelos de textura franca a arcillosa y reducido distanciamiento entre las hileras de vid. Es por ello que para la mayoría de los viñedos irrigados con la tecnología de riego localizado, la más difundida en la región, sería importante la utilización de especies herbáceas nativas como cultivos de cobertura. Por observaciones directas a campo, es creciente el número de viñedos en los que favorecen el establecimiento y resiembra espontánea de especies nativas. Los resultados de la evaluación de la cubierta vegetal en el sitio interfilar, en los tres viñedos bajo seguimiento, permitió verificar que se pueden alcanzar aceptables niveles de cobertura del suelo mediante el uso de herbáceas nativas (datos no presentados).

Mediciones de huella hídrica indican que distintas variedades de vid en Mendoza poseen un consumo hídrico similar, y que los factores de mayor incidencia en el cálculo son el nivel de producción y el sistema de riego (Conte \& Fayad, 2012; Morábito, 2012). Cuando la producción de uva es alta y el riego es eficiente, la huella hídrica es menor. Las importantes diferencias encontradas en el consumo de agua, para los diferentes cultivos de cobertura y las alternativas de manejo con suelo descubierto, determinan la importancia de tener en cuenta el tipo de manejo del suelo para futuras mediciones de huella hídrica en el viñedo.

\section{Dinámica de la Evt en el transcurso del año}

Al comparar la Evt media diaria para las diferentes épocas del año, se constataron valores similares de Evt para las diferentes especies en primavera y verano, teniendo en cuenta períodos sin restricción hídrica. En un verano con restricción hídrica, se registraron valores de Evt inferiores a los de primavera sin déficit de agua. La menor disponibilidad hídrica en el sustrato y el mayor estrés térmico de los típicos días de verano, pueden haber determinado una mayor intensidad en el cierre estomático (Cox \& Boersma, 1967), induciendo un menor consumo diario promedio.

Para un suelo descubierto sin restricción hídrica, hubo mayor evaporación en verano respecto de la medida en primavera; mientras que para un verano con déficit de agua, la evaporación fue inferior a la de primavera con suelo húmedo.

Se pudo verificar que durante un verano con restricción hídrica, las especies nativas $\mathrm{C}_{4}$ consumieron mayor o igual cantidad de agua en comparación con malezas $\mathrm{C}_{4}$ y perennes tipo $\mathrm{C}_{3}$, lo que se puede explicar por la capacidad de las plantas de desierto de mantener sus estomas abiertos en condiciones de estrés hídrico (Gibson, 1998). Mientras que en el transcurso de un verano sin limitación de agua, fueron las especies perennes $C_{3}$ y malezas $\mathrm{C}_{4}$ las que presentaron un consumo igual o superior al de las nativas (principalmente respecto de las tipo $\mathrm{C}_{4}$ ).

Durante primavera sin restricción hídrica las especies nativas $\left(\mathrm{C}_{4}\right.$ y $\left.\mathrm{C}_{3}\right)$ tuvieron un consumo igual o inferior al de malezas $\mathrm{C}_{4}$ y exóticas cultivadas $\mathrm{C}_{3}$. En tanto que en otoño e invierno las especies $C_{3}$ (i.e, exóticas cultivadas y nativa) mostraron una Evt superior a las especies $\mathrm{C}_{4}$, las que en este período se encuentran en reposo vegetativo.

\section{Tasas de intercambio gaseoso}

Durante días de verano sin restricción hídrica, las tasas de $A$ fueron en general mayores para las especies tipo $\mathrm{C}_{4}$, tanto malezas como nativas. En condiciones de sequía, las especies $\mathrm{C}_{4}$ redujeron en menor proporción su Evt en comparación con las especies tipo $\mathrm{C}_{3}$. Las tasas de fijación disminuyeron levemente para las especies tipo $\mathrm{C}_{4}$, mientras que las $\mathrm{C}_{3}$ presentaron tasas de $E$ de dióxido de C. Estas observaciones corroboran la afirmación de que muchas de las adaptaciones estructurales y fisiológicas de las plantas de desierto sirven 
para maximizar la fotosíntesis y no tanto para limitar la pérdida de agua, como se asumía años atrás (Gibson, 1998).

\section{Eficiencia en el uso del agua}

Los mayores valores de Eua se registraron con especies tipo $\mathrm{C}_{4}$ (i.e., nativas o malezas) en condiciones de restricción hídrica. El aumento en la Eua a medida que disminuye el nivel de humedad en el suelo, ha sido observado previamente (Singh \& Misra, 1985). Este aumento se explicó en el ensayo debido a que en situación de estrés hídrico, la Evt se redujo proporcionalmente más que la fijación de C.

A niveles de $A$ relativamente bajos, la Eua de las especies $\mathrm{C}_{4}$ (i.e, nativas y malezas) fue mayor en suelos con restricción hídrica. Con sustrato húmedo se requirieron altas tasas de $A$ para lograr elevada Eua.

Durante jornadas calurosas de verano las especies nativas $\mathrm{C}_{4}$ presentaron mayores tasas de $A$ y mayor Eua que las especies $C_{3}$, tanto nativas como exóticas cultivadas. Esto es debido a que las plantas $\mathrm{C}_{4}$ evitan las pérdidas de $\mathrm{C}$ por fotorespiración (Larcher, 2003). En dichas condiciones la mayor eficiencia se observó durante las horas de la mañana, cuando la Evt fue relativamente menor en comparación con la tarde, y la $A$ neta fue similar. Este comportamiento se explica por las temperaturas más moderadas que se registran durante el transcurso de la mañana.

Se comprobó la mayor actividad fotosintética neta de las especies $\mathrm{C}_{4}$ durante días calurosos, presentando un consumo hídrico similar al de especies tipo $\mathrm{C}_{3}$, pero con mayores niveles de $A$. Durante una jornada estival, una especie nativa tipo $\mathrm{C}_{4}$ alcanzó a asimilar hasta $4 \mathrm{~g} \mathrm{CO}_{2} \mathrm{~m}^{-2}$ día $^{-1}$, en comparación con una $E$ de 2 a $5 \mathrm{~g} \mathrm{CO}_{2} \mathrm{~m}^{-2}$ día $^{-1}$ generada por el suelo descubierto y una especie perenne tipo $\mathrm{C}_{3}$, respectivamente. Estas importantes diferencias en el balance de gases se atribuyen a una alta eficiencia fotosintética y baja respiración en especies con camino metabólico tipo $\mathrm{C}_{4}$ (Bull, 1969).

La mayor $E$ registrada por especies tipo $\mathrm{C}_{3}$, en comparación con el suelo descubierto, se puede explicar por un aumento en la tasa de respiración y una mayor actividad microbiana propia de un suelo con cobertura vegetal. Al respecto, Frank et al. (2002) atribuyeron el aumento del flujo de dióxido de $\mathrm{C}$ en el suelo a los mayores contenidos de carbón orgánico y carbono de biomasa microbiana, y sugirieron que el manejo del suelo juega un rol significativo en la respiración edáfica.

Las notables diferencias encontradas en el balance de $\mathrm{C}$ de las distintas coberturas verdes y su contraste respecto del manejo con suelo descubierto, revelan que resultaría apropiado tener en cuenta el tipo de gestión del suelo en el viñedo para obtener una aproximación más precisa a la huella de $\mathrm{C}$ de la uva. Aunque el protocolo internacional para el cálculo de $\mathrm{C}$ en la industria del vino considera el tipo de manejo del suelo, se reconoce allí que el potencial secuestro de $\mathrm{C}$ de los cultivos de cobertura no es del todo entendido hasta el momento y que se requiere disponer de información más precisa para su correcta interpretación (FIVS, 2008). Para poder estimar la potencial fijación de $C$ de los cultivos de cobertura es necesario obtener datos locales sobre el balance diario de C, en diferentes estaciones del año y bajo distintas condiciones ambientales.

\section{Limitaciones de la metodología del experimento}

Cabe considerar que los resultados fueron obtenidos con las especies vegetando en macetas, en un sustrato distinto a los suelos de la región y con una frecuencia de riego muy diferente al régimen de precipitaciones locales. Por lo tanto, los datos son útiles para comparar el comportamiento de las especies en condiciones controladas, pero deben ser validados con experiencias de campo y evaluando la interacción entre los cultivos de cobertura y la vid, bajo condiciones ecológicas locales.

\section{CONCLUSIONES}

La utilización de especies herbáceas nativas como cultivos de cobertura contribuye a reducir el consumo hídrico respecto del uso de coberturas vegetales cultivadas introducidas.

En condiciones de alta temperatura y restricción hídrica, las especies del tipo $\mathrm{C}_{4}$, tanto nativas como malezas, hacen un uso más eficiente del agua en comparación con las especies $\mathrm{C}_{3}$. La eficiencia está determinada por el grupo funcional al cual pertenecen, más que por su condición de planta nativa o exótica.

Especies nativas tipo $\mathrm{C}_{4}$ surgen con probabilidad de éxito como cultivos de cobertura el sitio interfilar de viñas bajo riego por goteo. Esto es debido a su capacidad de mantener mayor actividad de Evt bajo condiciones limitantes de agua disponible y su menor consumo hídrico anual.

Especies nativas de actividad invernal del tipo 
$\mathrm{C}_{3}$ (e.g., N. tenuis), con ciclo fenológico opuesto al del cultivo de la vid, pueden ser recomendadas en situaciones donde se busque reducir la excesiva competencia con el cultivo durante los meses de primavera.

Las mediciones de intercambio gaseoso de la planta entera mediante cámaras abiertas para canopia en cultivos de cobertura, originaron datos que pueden contribuir al desarrollo de modelos de balance del agua en el viñedo y de $A$ o $E$ de carbono para diversos fines.

\section{AGRADECIMIENTOS}

Parte del presente trabajo fue posible gracias a una beca otorgada por el Deutscher Akademischer Austausch Dienst (DAAD) y el Ministerio de Educación del Gobierno Argentino. Reconocimiento para el Dr. Prof. H. Schultz por su colaboración y aportes. Agradecimiento para el Ing. R. del Monte. Para V. Rodríguez, F. Ferrari, M. Bonada, M. Montoya, J. Guarise, A. Ambrogetti, A. Porro, P. Díaz y G. Montoya. Para A. Geib por sus sugerencias y a C. Frings por su apoyo técnico. A los Ings. Agrs. M. Ariza, S. Rinaldi y D. Dalla Torre, por su cooperación en las parcelas de seguimiento en viñedos de fincas comerciales, las cuales fueron puestas al servicio de la investigación. Al Dr. F. Casassa por la corrección del summary.

\section{BIBLIOGRAFÍA}

Boutraa, T., 2010. Improvement of water use efficiency in irrigated agriculture: a review. Journal of Agronomy, 9: 1-8.

Bowman, G., C. Shirley and C. Cramer, 1998. Managing cover crops profitably. USDA Sustainable Agriculture Network. Beltsville, MD. 212 pp.

Bull, T.A., 1969. Photosynthetic efficiencies and photorespiration in Calvin cycle and $\mathrm{C}_{4}$-dicarboxylic acid plants. Crop Science, 9(6): 726-729.

Catania, C.D., M.S. Avagnina De Del Monte, E.M. Uliarte, R.F. Del Monte y J. Tonietto, 2007. El clima vitícola de las regiones productoras de uvas para vinos de Argentina. En: Tonietto, J. y V. Sotés (Eds.).Caracterização climática de regiões vitivinícolas ibero-americanas. Bento Gonçalves, Brasil: CYTED, Embrapa, INTA.

Cavagnaro, J.B. y A.D. Dalmasso, 1986. Ensayo bajo riego de pasturas nativas en áreas cultivadas. Informe interno. Mendoza: IADIZA.

Celette, F., A. Findeling and C. Gary, 2009. Competition for nitrogen in an unfertilized intercropping system: The case of an association of grapevine and grass co- ver in a Mediterranean climate. European Journal of Agronomy, 30(1): 41-51.

Centinari, M., S. Poni, D.S. Intrigliolo, D. Dragoni and A.N. Lakso, 2012. Cover crop evapotranspiration in a northeastern US Concord (Vitis labruscana) vineyard. Australian Journal of Grape and Wine Research, 18(1): 73-79.

Conte, S. y F. Fayad, 2012. El agua que no se ve en lo que comemos. El caso de la uva mendocina. Diario Los Andes. Sociedad. Mendoza, 25 de marzo de 2012.

Cox, L.M. and L. Boersma, 1967. Transpiration as a function of soil temperature and soil water stress. Plant Physiology, 42(4): 550-556.

Del Monte, R.F., C.A. Mathey y M.E. Quiroga De Oriolani, 1994. Eficiencia comparativa entre sistemas de labranza y coberturas de flora natural en viticultura regadía. Horticultura Argentina, 13 (34-35): 87-98.

Del Monte, R.F., A. Ambrogetti, C. Catania, S. Avagnina De Del Monte y J. Perez Peña, 2000. Manejo de suelo mediante coberturas vegetales en el viñedo regadío. Resultados preliminares ciclo: 1999. Trabajo presentado en Encuentro con el Malbec, INTA EEA Mendoza.

Di Rienzo, J.A., F. Casanoves, M.G. Balzarini, L. Gonzalez, M. Tablada y C.W. Robledo, 2011. InfoStat. Córdoba, Argentina: Grupo InfoStat F.C.A. Universidad Nacional de Córdoba.

Donaldson, D.R., R.L. Snyder, C. Elmore and S. Gallagher, 1993. Weed control influences vineyard minimum temperatures. American Journal of Enology and Viticulture, 44(4): 431-434.

FIVS, 2008. International wine carbon calculator protocol. Version 1.2. Paris, France. $152 \mathrm{pp}$.

Frank, A.B., M.A. Liebig and J.D. Hanson, 2002. Soil carbon dioxide fluxes in northern semiarid grasslands. Soil Biology and Biochemistry, 34(9): 1235-1241.

Gibson, A.C., 1998. Photosynthetic organs of desert plants. BioScience, 48(11): 911-920.

Gulick, S.H., D.W. Grimes, D.A. Goldhamer and D.S. Munk, 1994. Cover-crop-enhanced water infiltration of a slowly permeable fine sandy loam. Soil Science Society of America Journal, 58(5): 1539-1546.

Hulme, M. y N. Sheard, 1999. Escenarios de cambio climático para Argentina. Norwich, Reino Unido: Unidad de Investigación Climática. 6 pp.

Larcher, W., 2003. Carbon utilization and dry matter production. 2 Pp. 69-173. En: Physiological plant ecology: ecophysiology and stress physiology of functional groups. 4th ed. Berlin-Heidelberg-New York: SpringerVerlag.

Lopes, C., A. Monteiro, F.E. Rückert, B. Gruber, B. Steinberg and H.R. Schultz, 2004. Transpiration of grapevines and co-habitating cover crop and weed species in a vineyard. A "snapshot" at diurnal trends. Vitis, 43(2): 
$111-117$.

Maigre, D., J. Aerny et F. Murisier, 1995. Entretien des sols viticoles et qualité des vins de Chasselas: influence de l'enherbement permanent et de la fumure azotée. Revue Suisse Vitiulture Arboriculture Horticulture, 27: 237-251.

Microsoft Corporation, 2010. Excel. Redmond, WA, USA.

Monteiro, A. and C.M. Lopes, 2007. Influence of cover crop on water use and performance of vineyard in Mediterranean Portugal. Agriculture, Ecosystems \& Environment, 121(4): 336-342.

Morábito, J., 2012. Las eficiencias de riego y la determinación de la huella hídrica en nuestros oasis regadíos. <http://www.imd.uncu.edu.ar/upload/morabito.pdf> Consultada el 12/04/2013.

Olmstead, M.A., R.L. Wample, S.L. Greene and J.M. Tarara, 2001. Evaluation of potential cover crops for inland pacific northwest vineyards. American Journal of Enology and Viticulture, 52(4): 292-303.

Penfold, C.M., 2010. Native grass cover crops. The Australian \& New Zealand Grapegrower \& Winemaker, (554): 48-51.

Prichard, T.L., W.M. Sills, W.K. Asai, L.C. Hendricks and C.L. Elmore, 1989. Orchard water use and soil characteristics. California Agriculture, 43(4): 23-25.

Sainju, U.M., B.P. Singh and W.F. Whitehead, 2002. Longterm effects of tillage, cover crops, and nitrogen fertilization on organic carbon and nitrogen concentrations in sandy loam soils in Georgia, USA. Soil and Tillage Research, 63(3-4): 167-179.

Sarrantonio, M., 1995. Leguminosas mejoradoras del suelo. Metodologías de evaluación. Instituto Rodale. Kutztown, EEUU. 301 pp.
Singh, K. and G. Misra, 1985. Water-use efficiency of one $\mathrm{C}_{3}$ and two $\mathrm{C}_{4}$ grasses in response to varying soil moisture and herbage-removal levels in a seasonally dry tropical region. Plant and Soil, 88(2): 171-180.

Spring, J. et N. Delabays, 2006. Essai d'enherbement de la vigne avec des espèces peu concurrentielles: aspects agronomiques. Revue suisse de viticulture arboriculture horticulture, 39(6): 355-359.

Systat Software Inc., 2008. Sigmaplot 11.0. Richmond, CA, USA.

Uliarte, E.M. y R.F. Del Monte, 2006. Coberturas de flora establecida en viticultura regadía. Informe final de convenio de asistencia técnica: INTA EEA Mendoza - Bodega Norton S.A. Luján de Cuyo: INTA EEA Mendoza. 54 pp.

Uliarte, E.M., R.F. Del Monte y C.A. Parera, 2006. Influencia del manejo de suelo mediante coberturas vegetales en el microclima de viñedos bajo riego (cv. Malbec). Le Bulletin de l'OIV, 79 (899-900): 5-22.

Uliarte, E.M., R.F. Del Monte, A.O. Ambrogetti y M.A. Montoya, 2009a. Evaluación y elección de diferentes especies de coberturas vegetales en viñedos bajo riego de Mendoza. Revista 13, año 2(15): 18-34.

Uliarte, E.M., R.F. Del Monte, C.A. Parera, C.D. Catania y S.M. Avagnina De Del Monte, 2009b. Influencia del manejo de suelo mediante coberturas vegetales establecidas en el desarrollo vegetativo, producción y características de vinos en viñedos bajo riego superficial (cv. Malbec). Le Bulletin de I'OIV, 82(938-939-940): 205-227.

Yunusa, I.A.M., R.R. Walker and J.R. Guy, 1997. Partitioning of seasonal evapotranspiration from a commercial furrow-irrigated Sultana vineyard. Irrigation Science, 18(1): 45-54. 